\title{
Size matters: Indexed left atrial size predicts late survival after aortic valve replacement
}

\author{
Richard J. Shemin, MD
}

\footnotetext{
From the Division of Cardiac Surgery, Department of Surgery, Cardiovascular Center at UCLA, David Geffen School of Medicine at UCLA, Los Angeles, Calif.

Disclosures: Author has nothing to disclose with regard to commercial support.

Received for publication Sept 5, 2017; accepted for publication Sept 13, 2017; available ahead of print Oct 7, 2017.

Address for reprints: Richard J. Shemin, MD, Ronald Reagan UCLA Medical Center, David Geffen School of Medicine at UCLA, 100 UCLA Medical Plaza, Suite 730, Los Angeles, CA 90095 (E-mail: Rshemin@ mednet.ucla.edu).

J Thorac Cardiovasc Surg 2018;156:600

$0022-5223 / \$ 36.00$

Copyright $\subset 2017$ Published by Elsevier Inc. on behalf of The American Association for Thoracic Surgery

https://doi.org/10.1016/j.jtcvs.2017.09.060
}

The cardiovascular community is in continuous search to identify the strongest independent predictors of late outcomes in our patients. Patients requiring an aortic valve replacement (AVR) have secondary physiologic changes in the left ventricle (LV), often leading to increases in LV end-diastolic and left atrial (LA) pressures. Enlargement of the LA is a secondary consequence of chronically elevated LA pressure and LV diastolic dysfunction. The measurement of LA size should be indexed for correct measurement and to account for body mass index and sex so that normal ranges and comparisons can be made.

In their study, "Indexed Left Atrial Size Predicts AllCause and Cardiovascular Mortality in Patients Undergoing Aortic Valve Surgery," Mosquera and colleagues ${ }^{1}$ found indexed LA size to be a strong independent predictor of cardiovascular mortality. Normal, mild, moderate, and severe LA enlargements, respectively, led to $97.6 \%, 86.8 \%$, $77.9 \%$, and $77.4 \%$ 5-year cardiovascular survivals $(P<.001)$. Indexed LA size did not affect operative mortality in this study. The enlarged LA size (indexed) had adverse survival consequences for both patients with aortic stenosis and patients with aortic insufficiency requiring an AVR. In addition, the enlarged LA was a strong independent predictor of all-cause mortality and of the combined event of stroke and cardiovascular mortality, but not of stroke alone.

Indexed LA size is easily measured by routine echocardiography. Enlargement of the LA can be caused by several factors, but in patients undergoing AVR, it is most commonly a consequence of LV diastolic dysfunction. It is associated with chronically elevated LA pressure. Surgeons often do not pay close attention to this measurement, and our databases minimize its importance. In addition, LA

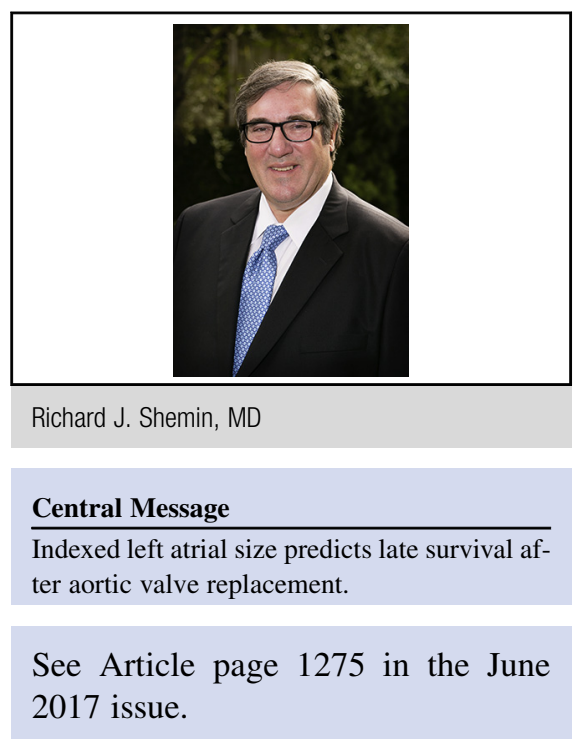

size has consequences for the development of atrial fibrillation and the consideration of a concomitant maze procedure. This study of Mosquera and colleagues ${ }^{1}$ emphasizes the independent predicative value of indexed LA size with respect to late cardiovascular mortality, all-cause mortality, and the combined event of stroke and cardiovascular mortality. We thus have a simple measure to help prognosticate the future of our patients, and perhaps to manage these patients better after AVR.

Most importantly, new research efforts should monitor the postoperative changes in indexed LA size to determine whether a return to normal or an improvement predicts improved survival. In addition, we have developed criteria to operate on symptom-free patients with aortic valve disease on the basis of LV function (ejection fraction) and LV size criteria. Perhaps enlarged LA size in symptomfree patients with aortic valve disease should be an additional indication for AVR.

\footnotetext{
Reference

1. Mosquera VX, Bouzas-Mosquera A, González-Barbeito M, BautistaHernandez V, Muñiz J, Alvarez-García N, et al. Indexed left atrial size predicts all-cause and cardiovascular mortality in patients undergoing aortic valve surgery. J Thorac Cardiovasc Surg. 2017; 153:1275-84.e7.
} 